psychopraxis. neuropraxis $2021 \cdot 24: 242-246$ https://doi.org/10.1007/s00739-021-00737-w Angenommen: 24. Juni 2021

Online publiziert: 13. Juli 2021

(๑) Der/die Autor(en) 2021

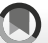

Andreas Erfurth ${ }^{1,3,4} \cdot$ Nikolaus Michael $^{2,4} \cdot$ Cornelia Roestel $^{4} \cdot$ Gabriele Sachs $^{3}$

'1. Abteilung für Psychiatrie und Psychotherapeutische Medizin, Klinik Hietzing, Wien, Österreich ${ }^{2}$ Klinik für Psychiatrie, Psychotherapie und Psychosomatik, Krankenhaus Elbroich, Düsseldorf, Deutschland ${ }^{3}$ Medizinische Universität Wien, Wien, Österreich

${ }^{4}$ Klinik für Psychiatrie und Psychotherapie, Universitätsklinikum Münster, Münster, Deutschland

\title{
Informationen zu bipolaren Störungen und Darstellungen in Film und Literatur
}

Bewältigung von Depressionen: https:// www.depressionen.ch), sowie die Depression and Bipolar Support Alliance (DBSA: https://www.dbsalliance.org).

In zahlreichen Büchern können Patientinnen und Patienten Informationen über Diagnose und Therapie erhalten, empfehlenswert sind unter anderem:

- Schou, Mogens. Die Lithiumtherapie affektiver Störungen: Praktische Informationen für Ärzte, Patienten und Angehörige. Thieme; 6., überarbeitete
Auflage (8. Dezember 2004) ISBN13: 978-3135933061.

- Bräunig, Peter. Leben mit bipolaren Störungen: Manisch-depressiv: Antworten auf die meistgestellten Fragen. TRIAS; 3. Auflage (13. Juni 2018) ISBN-13: 978-3432105789

- Meyer, Thomas D. und Hautzinger, Martin. Ratgeber Manisch-depressive Erkrankung: Informationen für Menschen mit einer bipolaren Störung und deren Angehörige. Hogrefe Verlag; piert, hier können unbeantwortet gebliebene Fragen im Detail beantwortet werden. In einigen Gruppen wurden rechtliche Aspekte angesprochen (z. B. das Unterbringungsgesetz). A Abb. 1 zeigt die Inhalte des Moduls.

》) Selbsthilfe stellt für bipolare

Personen ein sehr wichtiges Konzept dar

Hilfreich und von vielen Teilnehmern gewünscht ist die weitere Auseinandersetzung mit dem Thema der eigenen Bipolarität - über die Teilnahme an der psychoedukativen Gruppe hinaus. Wichtig ist die Vermittlung von lokalen Adressen der Selbsthilfe als auch von jenen Institutionen, die sich seit vielen Jahren international für die Belange bipolarer Menschen einsetzen. Hier sind vor allem zu nennen: die Deutsche Gesellschaft für bipolare Störungen (https://dgbs. de), die 2003 erstmals ein „Weißbuch Bipolare Störungen in Deutschland“ [3] herausgegeben hat, der in der Schweiz tätige Verein „Equilibrium“ (Verein zur

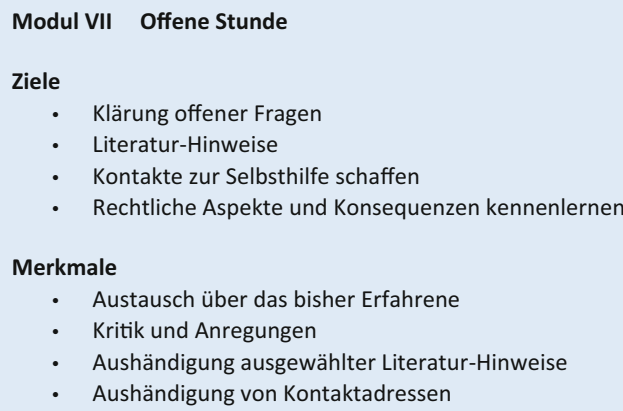

Abb. $1<$ Inhalte des Moduls VII 
1. Auflage 2013 (9. Juli 2013). ISBN13: 978-3801725198.

Eine Besonderheit im bipolaren Spektrum spielt die cyclothyme Störung, leider ist der wichtigste Ratgeber für Patientinnen und Patienten (noch) nicht ins Deutsche übersetzt worden: Hantouche, Elie; Majdalani, Caline und Blain, Régis. J'apprends à gérer ma cyclothymie (Französisch), Josette Lyon (7. September 2012) ISBN-13: 978-2843192722.

Aufschlussreich können Bücher von Betroffenen sein, so die Darstellungen des eigenen bipolaren Verlaufes in:

- Melle, Thomas. Die Welt im Rücken. Rowohlt Taschenbuch; 5. Auflage (20. Februar 2018). ISBN-13: 9783499272943 sowie in Taschenbuch 20. April 2021

- Hornbacher, Marya. Gefangen in meinem Selbst: Mein Leben mit einer bipolaren Störung. mvg Verlag (20. April 2021). ISBN-13: 9783747402818

Besonders empfehlenswert sind die Bücher von Kay Redfield Jamison, die teilweise auch in deutscher Sprache vorliegen:

- Meine ruhelose Seele: Die Geschichte einer bipolaren Störung (Deutsch). mvg Verlag; 1. Auflage (15. August 2014). ISBN-13: 978-3868825046

- Wenn es dunkel wird. Zum Verständnis des Selbstmordes (Deutsch). Berliner Taschenbuchverlag (1. Januar 2002). ISBN-13: 978-3442760886

- Exuberance: The Passion for Life (Englisch). Vintage; Reprint Edition (13. September 2005) Taschenbuch. ISBN-13: 978-0375701481

- Touched With Fire: Manic-Depressive Illness and the Artistic Temperament (Englisch). Free Press; Reissue Edition (18. Oktober 1996) ISBN-13: 9780684831831

In vielfältiger Weise wurden bipolare Störungen in Literatur und Film dargestellt. Als empfehlenswerte Beschreibungen in der Literatur seien genannt:

- Eugenides, Jeffrey. Die Liebeshandlung (The Marriage Plot) Rowohlt; 1. Auflage (18. Oktober 2011) ISBN13: 978-3498016746. Der Roman
Hier steht eine Anzeige.

Springer 
beschreibt eine Dreiecksgeschichte im Milieu einer amerikanischen Elite-Universität. Zwei Kommilitonen bemühen sich um die Liebe der Studentin Madeleine: der bipolare Leonhard und Mitchell, der autobiographische Züge trägt.

- Widmer, Urs. Der Geliebte der Mutter. Diogenes; 1. Auflage (25. September 2012) ISBN-13: 978-3257057324.

In dieser (fiktiven) Autobiographie beschreibt Widmer die an einer bipolaren Störung leidende Mutter aus der Perspektive des Kindes.

- Haslett, Adam. Stellt euch vor, ich bin fort (Imagine Me Gone) Rowohlt; 1. Auflage (24. Januar 2018) ISBN13: 978-3498030285. Der Roman stellt die Geschichte einer bipolaren Familie über zwei Generationen dar.

\section{1) Zahlreich sind die Darstellungen bipolarer Störungen in Literatur und Film}

Das Genre Film ist besonders gut geeignet, um verschiedene Facetten affektiver Störungen darzustellen. Folgende Filme seien als Beispiele genannt.

Einen depressiven Mischzustand zeigt: Von Angesicht zu Angesicht (Ansikte mot ansikte). Ingmar Bergman, Schweden 1976 mit Liv Ullmann, Erland Josephson, Gunnar Björnstrand und Aino Taube.

Darstellungen der Manie finden sich in:

- Aguirre, der Zorn Gottes. Werner Herzog, Deutschland, Mexiko, Peru 1972 mit Klaus Kinski, Helena Rojo und Ruy Guerra,

- Eine Frau unter Einfluß (A Woman under the Influence). John Cassavetes, USA 1974 mit Gene Rowlands und Peter Falk,

- Mr. Jones. Mike Figgis, USA 1993 mit Richard Gere, Lena Olin und Anne Bancroft.

In einigen Filmen ist die bipolare Störung nicht der zentrale Handlungsstrang, sondern mehr die Kulisse für eine tragikomische Erzählung, so in Silver Linings (Silver Linings Playbook). David O. Russell,
USA 2012 mit Bradley Cooper, Jennifer Lawrence und Robert De Niro sowie in Infinitely Polar Bear. Maya Forbes, USA 2014 mit Mark Ruffalo, Zoë Saldaña und Keir Dullea.

Eine besondere Bedeutung für bipolare Verläufe hat das hyperthyme Temperament im Übergang zur Hypomanie. Für Patientinnen und Patienten ist es wichtig zu erkennen, dass die Hypomanie nicht nur eine „sonnige“ Seite haben kann [4-7], sondern auch eine „dunkle“ [5-7]; Hypomanien im Verlauf also nicht unbedingt immer erstrebenswert sind. Besonders beeindruckend ist in diesem Zusammenhang die Darstellung des hypomanen Bruno in Il sorpasso (deutscher Verleihtitel: Verliebt in scharfe Kurven). Dino Risi, Italien 1962 mit Vittorio Gassman, Jean Louis Trintignant und Catherine Spaak. Der auch im richtigen Leben bipolare Schauspieler Vittorio Gassman spielt den Bruno als einen mitreißenden, selbstsicheren, energetischen, verführerischen, aber moralisch verantwortungslosen und oberflächlichen Menschen. Es wird deutlich, dass der Bipolare sozial isoliert und tragisch einsam sein kann, sich die (Hypo)manie als Konzept für eine „Lebensphilosophie“ [8] letztlich doch „falsch“ anfühlt.

Im Modul VIII wird die retrospektive Life-Chart $[9,10]$ erlernt. - Abb. 2 zeigt die Inhalte des Moduls. Als Hausaufgabe für die nächsten Wochen soll für jedes vergangene Lebensjahr von jedem Teilnehmer der in $\mathbf{D} \mathbf{A b b} \mathbf{3}$ gezeigte Life-Chart-Bogen ausgefüllt werden. Das ermöglicht gemeinsam mit der bereits erlernten prospektiven Life-Chart $[1,9,10]$ eine lückenlose Erfassung des Verlaufes $[2,11]$ und seiner Determinanten [12-17] durch die bipolare Person. Manche Aspekte des Moduls V (Verlauf) werden durch das Ausfüllen der retrospektiven Life-Chart am eigenen Beispiel deutlich, etwa die Bedeutung von Mischzuständen [18, 19], von Komorbiditäten [20-27], von Polaritätswechseln [28, 29], von Therapieresistenz [29-31] und des Verlaufstyps [32, 33]. Dieses stellt eine große Hilfe für den therapeutischen Prozess dar - auch in Hinblick auf das Erreichen von Adhärenz [34] und einer guten psychosozialen Funktionsfähigkeit [35] - und macht die Patientin/den Pati- psychopraxis. neuropraxis $2021 \cdot 24$ :

242-246

https://doi.org/10.1007/s00739-021-00737-w (c) Der/die Autor(en) 2021

A. Erfurth - N. Michael · C. Roestel · G. Sachs Informationen zu bipolaren Störungen und Darstellungen in Film und Literatur

\section{Zusammenfassung}

Hiermit veröffentlicht $p s y c h o p r a-$ xis.neuropraxis die dritte Tranche eines Psychoedukationsprogramms mit 8 Modulen. Vorgestellt wird eine offene Stunde, in der für das Konzept von Selbsthilfegruppen geworben wird und Anregungen zu Büchern mit Informationen gegeben werden, auch zu Darstellungen affektiver Störungen in Literatur und Film. In einem weiteren Modul lernen die Teilnehmer die retrospektive LifeChart kennen. Durch die Verknüpfung von retrospektiver mit prospektiver Life-Chart entsteht eine lückenlose Erfassung des Verlaufes und seiner Determinanten durch die bipolare Person.

Schlüsselwörter

Psychoedukation - Life-Chart-Methode . Literatur · Film

\section{Information on Bipolar Disorders and} Representations in Film and Literature

Abstract

Herewith psychopraxis.neuropraxis publishes the third tranche of a psychoeducation programme with 8 modules. An open hour is presented, promoting the concept of self-help groups and suggesting books with information, including portrayals of affective disorders in literature and film. In another module, participants learn about the retrospective life chart. By linking retrospective with prospective life charting, a complete record of the course and its determinants is created by the bipolar person.

Keywords

Psychoeducation - Life chart method . Literature · Film 


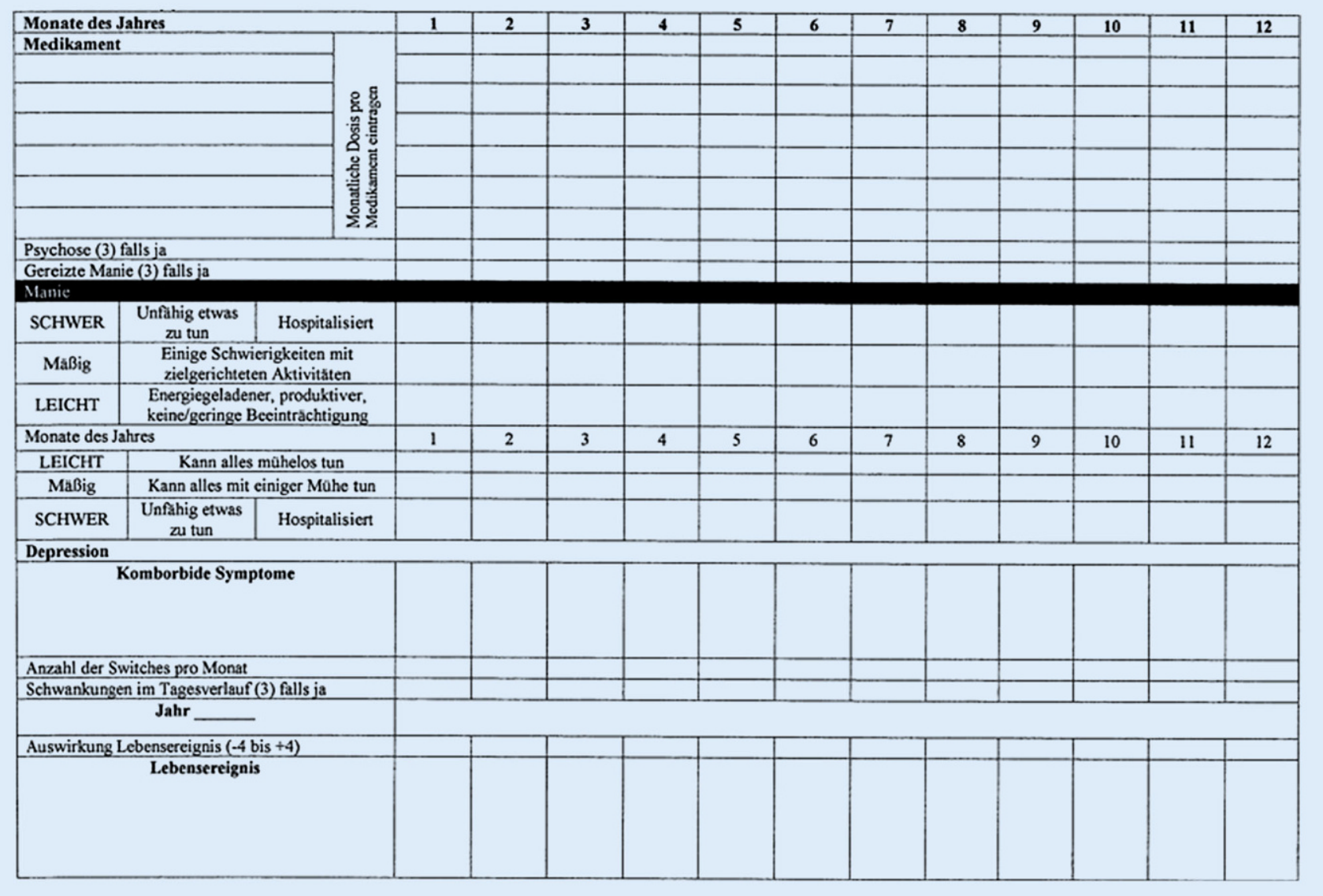

Abb. $3 \Delta$ Retrospektive Life-Chart. (Nach Leverich und Post [9] und Dittmann et al. [10])

enten zur Expertin/zum Experten ihrer/ seiner Erkrankung.

Am Ende der Psychoedukation sollte jede Person alle Abbildungen der 3 Tranchen als Fotokopie erhalten haben; diese Sammlung kann ihr auch in der Zukunft als Gedächtnisstütze und für das Ausfüllen der prospektiven Life-Chart dienen.

\section{Fazit für die Praxis}

\section{- Psychoedukation ist ein wichtiger Bestandteil der Therapie bipolarer Störungen.}

- Die Zeitschrift psychopraxis.neuropraxis hat - über 3 Hefte verteilt - eine psychoedukative Intervention mit 8 Modulen abgedruckt.

- Die retrospektive Life-Chart ermöglicht die Erfassung des bisherigen bipolaren Verlaufes.

- Ziel von Psychoedukation ist, dass bipolare Personen eine eigene Ex- pertise über ihren Krankheitsverlauf erlangen.

Korrespondenzadresse
Andreas Erfurth
1. Abteilung für Psychiatrie
und Psychotherapeutische
Medizin, Klinik Hietzing
Wolkersbergenstr. 1,
1130 Wien, Österreich
andreas.erfurth@
gesundheitsverbund.at

Danksagung. Herrn Mag. Clemens Stampf (freier Journalist und Filmkritiker in Wien) sei gedankt für wertvolle Diskussion und Hinweise im Zusammenhang mit Film und Literatur.

Funding. Open access funding provided by Medical University of Vienna.

\section{Einhaltung ethischer Richtlinien}

Interessenkonflikt. A. Erfurth, N. Michael, C. Roestel und G. Sachs geben an, dass kein Interessenkonflikt besteht.

Für diesen Beitrag wurden von den Autoren keine Studien an Menschen oder Tieren durchgeführt. Für die aufgeführten Studien gelten die jeweils dort angegebenen ethischen Richtlinien.

Open Access. Dieser Artikel wird unter der Creative Commons Namensnennung 4.0 International Lizenz veröffentlicht, welche die Nutzung, Vervielfältigung Bearbeitung, Verbreitung und Wiedergabe in jeglichem Medium und Format erlaubt, sofern Sie den/die ursprünglichen Autor(en) und die Quelle ordnungsgemäß nennen, einen Link zur Creative Commons Lizenz beifügen und angeben, ob Änderungen vorgenommen wurden.

Die in diesem Artikel enthaltenen Bilder und sonstiges Drittmaterial unterliegen ebenfalls der genannten Creative Commons Lizenz, sofern sich aus der Abbildungslegende nichts anderes ergibt. Sofern das betreffende Material nicht unter der genannten Creative Commons Lizenz steht und die betreffende Handlung nicht nach gesetzlichen Vorschriften erlaubt ist, ist für die oben aufgeführten Weiterverwendungen des Ma- 
terials die Einwilligung des jeweiligen Rechteinhabers einzuholen.

Weitere Details zur Lizenz entnehmen Sie bitte der Lizenzinformation auf http://creativecommons.org/ licenses/by/4.0/deed.de.

\section{Literatur}

1. Erfurth A, Michael N, Roestel C, Sachs G (2021) Psychoedukation für bipolare Störungen - die prospektive Life-Chart-Methode. Psychopraxis Neuropraxis 24:84-97. https://doi.org/10.1007/ s00739-020-00689-7

2. Erfurth A, Michael N, Roestel C, Sachs G (2021) Verlauf und Therapie bipolarer Störungen. Module für die Gruppenpsychoedukation. Psychopraxis Neuropraxis 24:156-165. https://doi.org/10.1007/ s00739-021-00718-z

3. Erfurth A (2003) Weißbuch Bipolare Störungen in Deutschland. Deutsche Gesellschaft für Bipolare Störungen e. V., Hamburg

4. Gamma A, Angst J, Ajdacic-Gross V, Rössler W (2008) Are hypomanics the happier normals? J Affect Disord 111(2-3):235-243. https://doi.org/ 10.1016/j.jad.2008.02.020

5. Akiskal HS, HantoucheEG, Allilaire JF (2003) Bipolar II with and without cyclothymic temperament: "dark" and "sunny" expressions of soft bipolarity. J Affect Disord 73(1-2):49-57. https://doi.org/10. 1016/s0165-0327(02)00320-8

6. Brand S, Gerber M, Pühse U, Holsboer-Trachsler E (2011) 'Bright side' and 'dark side' hypomania are associated with differences in psychological functioning, sleep and physical activity in a nonclinical sample of young adults. J Affect Disord 131(1):68-78. https://doi.org/10.1016/j.jad.2010. 12.007

7. Erfurth A, Dobmeier G, Zechendorff M (2005) Kurzpsychoedukation für bipolare Patienten. Thieme, Stuttgart

8. Hadot P (1995) Philosophy as a way of life. Blackwell, Malden

9. Leverich GS, Post RM (1996) Life charting the course of bipolar disorder. Curr Rev Mood Anxiety Disord 1:48-61

10. Dittmann S, Forsthoff A, Hummel B, Leverich GS (2001) Die Life-Chart-Methodik zur Erfassung des Langzeitverlaufs bipolarer Störungen. Nervenheilkunde 20(Suppl 2):25-29

11. Erfurth A, Arolt V (2003) Das Spektrum bipolarer Störungen. Nervenarzt 74(1):55-71. https://doi. org/10.1007/s00115-003-1473-x

12. Erfurth A, Gerlach AL, Hellweg I, Boenigk I, Michael N, Akiskal HS (2005) Studies on a German (Münster) version of the temperament auto-questionnaire TEMPS-A: construction and validation of the briefTEMPS-M. J Affect Disord 85(1-2):53-69. https://doi.org/10.1016/S0165-0327(03)00102-2

13. Erfurth A, Gerlach AL, Michael N, Boenigk I, Hellweg I, Signoretta S, Akiskal K, Akiskal HS (2005) Distribution and gender effects of the subscales of a German version of the temperament autoquestionnaire briefTEMPS-M in a university student population. J Affect Disord 85(1-2):71-76. https://doi.org/10.1016/j.jad.2003.07.003

14. Hantouche EG, Erfurth A (2010) Rethinking subthreshold bipolarity: the rebirth of KahlbaumHecker syndrome. Eur Psychiatr 25(S1):170. https://doi.org/10.1016/S0924-9338(10)70170-X

15. Erfurth A, Sachs G, Perugi G, Tondo L (2014) Athanasios Koukopoulos (1931-2013). Zum Verlauf manisch-depressiver Erkrankungen. Psychopra- xis Neuropraxis 17:5-8. https://doi.org/10.1007/ s00739-014-0172-7

16. Carta MG, Colom F, Erfurth A, Fornaro M, Grunze H Hantouche E, Nardi AE, Preti A, Vieta E, Karam E (2021) In memory of Hagop Akiskal. Clin Pract Epidemiol Ment Health 17:48-51. https://doi.org/ 10.2174/1745017902117010048

17. Kasper $S$, Baranyi $A$, Eisenburger $P$, Erfurth $A$, ErtI M, Frey R, Hausmann A, Kapfhammer HP, Roitner-Vitzthum E, Winkler D (2013) Konsensus-Statement: Die Behandlung der Agitation beim psychiatrischen Notfall. CliniCum neuropsy Sonderausgabe November, S3-15

18. Erfurth A, Sachs G (2015) Mischzustände, Mischbilder und die depressive Episode mit gemischten Merkmalen. Psychopraxis Neuropraxis 18(2):38-43. https://doi.org/10.1007/s00739015-0241-6

19. Tortorella A, Albert U, Nivoli AMA, Erfurth A, ColomF, Maina G(2015) Mixed states: stilla modern psychopathological syndrome? J Psychopathol 21:332-340

20. Shahini M, Shala M, Xhylani P, Gashi S, Borinca I, Erfurth A (2018) Challenging predictions between affective temperaments, depression and anxiety in a Kosovo student community sample. Int J Psychiatry Clin Pract 22(4):282-288. https://doi. org/10.1080/13651501.2018.1426771

21. Erfurth A, Sachs G (2020) Angststörungen. In: Steiner M (Hrsg) Angst. WAS, Bd. 113. Leykam, Graz, Wien

22. Sachs G, Erfurth A (2018) Obsessive compulsive and related disorders: from the biological basis to a rational pharmacological treatment. Int $J$ Neuropsychopharmacol 21(1):59-62. https://doi. org/10.1093/ijnp/pyx101

23. Vyssoki B, Blüml V, Gleiss A, Friedrich F, Kogoj $D_{\text {, }}$ Walter H, Zeiler J, Höfer P, Lesch OM, Erfurth A (2011) The impact of temperament in the course of alcohol dependence. J Affect Disord 135(1-3):177-183. https://doi.org/10.1016/j.jad. 2011.07.007

24. Skala K, Riegler A, Erfurth A, Völkl-Kernstock $S$ Lesch OM, Walter H (2016) The connection of temperament with ADHD occurrence and persistence into adulthood-An investigation in 18 year old males. J Affect Disord 198:72-77. https://doi.org/10.1016/j.jad.2016.03.051

25. Perugi G, Quaranta G, Belletti S, Casalini F, Mosti N, Toni C, Dell'Osso L (2015) General medical conditions in 347 bipolar disorder patients: clinical correlates of metabolic and autoimmune-allergic diseases. J Affect Disord 170:95-103. https://doi. org/10.1016/j.jad.2014.08.052

26. Erfurth A, Kuhn G (2000) Topiramate monotherapy in the maintenance treatment of bipolar I disorder: effects on mood, weight and serum lipids. Neuropsychobiology 42(Suppl 1):50-51. https:// doi org/10.1159/000054854

27. Perugi G, Erfurth A, Rihmer Z, Maremmani I, Brieger $P$ (2005) Comorbidity in bipolar disorders. Eur Psychiatr 20(S1):S120. https://doi.org/10. 1016/S0924-9338(05)80074-4

28. Reginaldi D, Tondo L, Caliari B, Minnai GP Kukopulos A (1982) The role of antidepressants in rapid cyclicity. Adv Biochem Psychopharmacol 32:363-367

29. Erfurth A, Perugi G (2005) Rapid cycling. In: Kasper S, Hirschfeld RMA (Hrsg) Handbook of bipolar disorder. Taylor \& Francis, New York, London, S 201-212

30. Reginaldi D, Tondo L, Floris G, Pignatelli A, Kukopulos A (1981) Poor prophylactic lithium response due to antidepressants. Int Pharma- copsychiatry 16(2):124-128. https://doi.org/10. 1159/000468484

31. Erfurth A, Möller HJ (2000) Vorgehen bei Antidepressiva-Nonrespondern. In: Möller HJ (Hrsg) Therapie psychiatrischer Erkrankungen, 2. Aufl. Thieme, Stuttgart, S407-413

32. Kukopulos A, Reginaldi D, Laddomada P, Floris G, Serra G, Tondo L (1980) Course of the manic-depressive cycle and changes caused by treatment. Pharmakopsychiatr Neuropsychopharmakol 13(4):156-167. https://doi.org/10.1055/s2007-1019628

33. Koukopoulos A, Reginaldi D, Tondo L, Visioli C, Baldessarini RJ (2013) Course sequences in bipolar disorder: depressions preceding or following manias or hypomanias. J Affect Disord 151(1):105-110. https://doi.org/10.1016/j.jad. 2013.05.059

34. Erfurth $A$ (2018) How can we improve adherence to treatment in bipolar disorders? Eur Psychiatr 48:31. https://doi.org/10.1016/j.eurpsy.2017.12.012

35. Sachs $G$, Berg $A$, Jagsch $R$, Lenz $G$, Erfurth $A$ (2020) Predictors of functional outcome in patients with bipolar disorder: effects of cognitive psychoeducational group therapy after 12 months. Front Psychiatry 11:530026. https://doi.org/10. 3389/fpsyt.2020.530026

Hinweis des Verlags. Der Verlag bleibt in Hinblick auf geografische Zuordnungen und Gebietsbezeichnungen in veröffentlichten Karten und Institutsadressen neutral. 